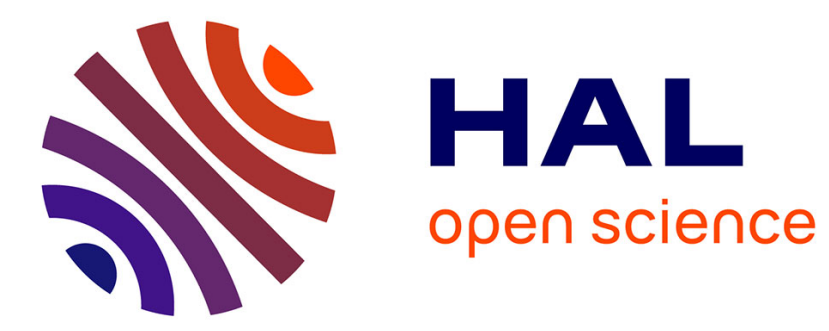

\title{
Non linear pattern selection in a problem of elasticity
}

Y. Pomeau

\section{To cite this version:}

Y. Pomeau. Non linear pattern selection in a problem of elasticity. Journal de Physique Lettres, 1981, 42 (1), pp.1-4. 10.1051/jphyslet:019810042010100 . jpa-00231862

\section{HAL Id: jpa-00231862 https://hal.science/jpa-00231862}

Submitted on 1 Jan 1981

HAL is a multi-disciplinary open access archive for the deposit and dissemination of scientific research documents, whether they are published or not. The documents may come from teaching and research institutions in France or abroad, or from public or private research centers.
L'archive ouverte pluridisciplinaire HAL, est destinée au dépôt et à la diffusion de documents scientifiques de niveau recherche, publiés ou non, émanant des établissements d'enseignement et de recherche français ou étrangers, des laboratoires publics ou privés. 


\title{
LE JOURNAL DE PHYSIQUE-LETTRES
}

03.40D

\section{Non linear pattern selection in a problem of elasticity}

\author{
Y. Pomeau \\ Service de Physique Théorique, CEN Saclay, B. P. No 2, 91190 Gif-sur-Yvette, France
}

(Reçu le 11 août 1980, révisé le 13 novembre, accepté le 14 novembre 1980)

\begin{abstract}
Résumé. - Le flambage de longues plaques rectangulaires offre la possibilité de tester une proposition récente. Le nombre d'onde des structures cellulaires dans des conditions faiblement supercritiques est déterminé par les conditions aux limites. Dans le cas présent, on prédit une décroissance supercritique de la longueur d'onde.
\end{abstract}

\begin{abstract}
The buckling of long rectangular elastic plates offers the possibility of testing a recent proposal. The wavenumber of cellular structures in slightly supercritical conditions is determined by the boundary conditions. In the present case a supercritical decrease of the wavelength is predicted.
\end{abstract}

Having in mind the wavelength selection in cellular flows, as in Rayleigh-Bénard convection in large horizontal layers, S. Zaleski and the author [1] solved the question of non linear pattern selection in slightly supercritical conditions for one dimensional models.

This sort of problem is formulated as follows : space dependent fluctuations with a (horizontal) fixed wavenumber, say $q_{0}$, become linearly unstable around a homogeneous rest state whenever a control parameter, say $\varepsilon$, exceeds some critical value, which can be taken at $\varepsilon=0$. The growth of these fluctuations is limited by non linear effects and a new steady state is reached via a supercritical (or normal) bifurcation. In a large class of problems, for slightly positive values of $\varepsilon$ linearly unstable fluctuations grow from the homogeneous state whenever their wavenumber belongs to a band of width of order $\varepsilon^{1 / 2}$ near the threshold value $q_{0}$. However, owing to the boundary conditions limiting the lateral extent of the structure, the supercritical steady pattern has its wavenumber in a much narrower band of width of order $\varepsilon$ near $q_{0}$.

The applicability of this sort of consideration to the Rayleigh-Bénard problem is not obvious, since long rolls, when parallel to a lateral boundary, are unstable against a cross roll instability localized near this boundary [1]. One should account for the structure of these boundary rolls parallel to their axis. This structure is due either to the cross roll modulation or to lateral boundaries inhibiting their growth. A realistic treatment of this problem is not an easy task.

Therefore it is of interest to look at a physical situation involving a non linear selection of the wavelength, but nevertheless permitting quantitative pre- dictions from simple $a b$ initio calculations. That is why I have considered the following version of the von Kármán problem in elasticity of thin plates [2].

This is the buckling of long rectangular elastic plates [3] submitted to a load along their long axis (see Fig. 1). According to the general considerations of reference [1], whenever the length of the long axis, say $L$, is much larger than a quantity of order $\varepsilon^{-1}$, one may limit oneself to the consideration of a half infinite problem. The possible buckling patterns for a large (but finite) $L$ are obtained by gluing together two half infinite solutions in a convenient way. We shall not consider this specific problem here; it is treated in reference [1].

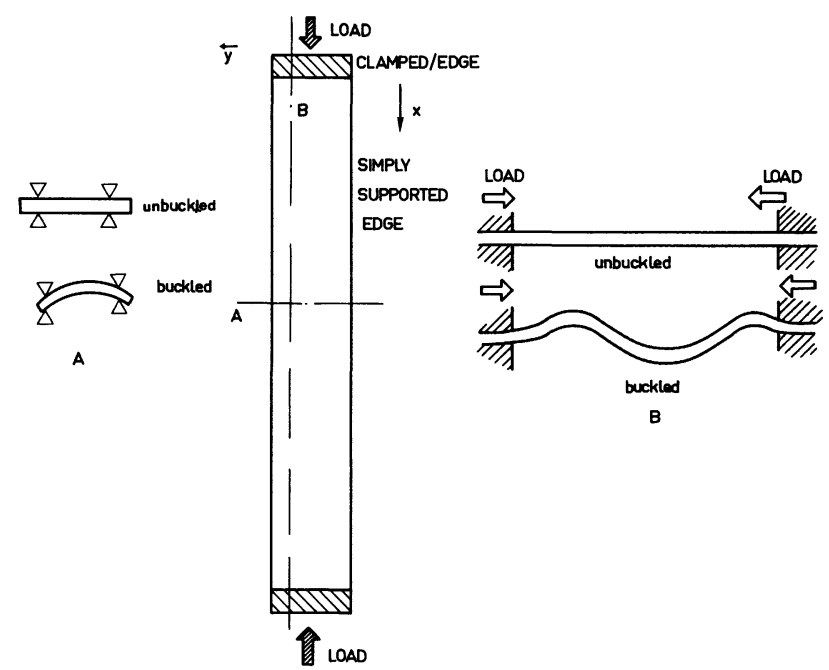

Fig. 1. - At the centre of the figure the plate is represented from above ; its cuts along the short $(A)$ and long $(B)$ are represented on the left and right. A similar figure is in reference [4]. 
The von Kármán [2] equations for the static buckling are $[4,5]$ :

$$
\begin{aligned}
\Delta^{2} w & =[\varphi, w]-\lambda w_{x x} \\
\Delta^{2} \varphi & =-\frac{1}{2}[w, w]
\end{aligned}
$$

where $w$ is the displacement of the plate perpendicular to its surface, $\varphi$ is the Airy stress function [5] and $\lambda$ is the load (and thus the control parameter in this problem). Moreover, $x$ and $y$ are the coordinates on the plate surface, respectively along the long and short axes of the plate, and

$$
[u, v] \equiv u_{x x} v_{y y}+u_{y y} v_{x x}-2 u_{x y} v_{x y}
$$

where $u_{x x}=\frac{\partial^{2} u}{\partial x^{2}}, \quad u_{x y}=\frac{\partial^{2} u}{\partial x y}, \ldots$. Finally the square Laplacian in $(1 a)$ and $(1 b)$ is taken in the plane of the plate : $\Delta^{2} \equiv \partial_{x^{4}}^{4}+2 \partial_{x^{2} y^{2}}^{4}+\partial_{y^{4}}^{4}$.

The boundary conditions (b.c.) can be taken as $w=\Delta w=0$ at the lateral boundary $(y= \pm h / 2$, $h$ being the width of the plate), this corresponding to the simply supported case [4]. The b.c. at the clamped edges (along the short edges of the plate, denoted as $\mid \partial$ from now on) are $\left.w\right|_{\partial}=\left.w_{x}\right|_{\partial}=0$. The simply supported b.c. make the problem easily manageable and are nevertheless realistic. The b.c. for $\varphi$ are [4] $\varphi_{\mathrm{N}}=(\Delta \varphi)_{\mathrm{N}}$, where the subscript $\mathrm{N}$ indicates differentiation in the normal direction (which yields $\varphi_{y}=\varphi_{y^{3}}=0$ along the long sides, and

$$
\left.\varphi_{x}\right|_{\partial}=\left.\varphi_{x^{3}}\right|_{\partial}=0
$$

along the short sides).

For an infinitely long plate, the $\varphi=w=0$ solution of (1) (this is the homogeneous rest state) bifurcates normally as $\lambda$ increases beyond $\lambda_{\mathrm{c}} \equiv 4 b^{2}$ where $b=\pi / h$ ( $h$ is the constant width of the plate), the corresponding linear perturbation being

$\varphi=0 \quad$ and $\quad w=w_{0} \sin a x \cos b y \quad$ with $a=b$.

The non linear Landau analysis of this bifurcation is straightforward and gives, for $\lambda \gtrsim \lambda_{\mathrm{c}}$, solutions of (1) with a small finite amplitude. The general method for computing explicitly this sort of solution has been outlined by Sorokin [6] for the Rayleigh-Bénard case and need not be explained here.

The result of this weakly non linear analysis is, near $\lambda=\lambda_{\mathrm{c}}$ and $a=b$ :

$$
\varphi \simeq \frac{w_{0}^{2}}{32}(\cos 2 a x-\cos 2 b y)
$$

$w \simeq w_{0} \sin a x \cos b y$ with $w_{0}^{2} \simeq \frac{8}{b^{2}}\left(\varepsilon-4 \delta^{2}\right)$

where $a \equiv b+\delta, \lambda \equiv \lambda_{\mathrm{c}}+\varepsilon, \delta$ and $\varepsilon$ both being small such that $\varepsilon \geqslant 4 \delta^{2}$. Higher order terms are neglected.
To deduce $\varphi$ from $(1 b)$, once $w$ is known, one is free to add to $\varphi$ an arbitrary quantity proportional to $y^{2}$. This only amounts to changing the load $\lambda$.

To find the possible wavenumbers for half infinite solutions with a clamped short edge, one follows a method very similar to that of the authors of reference [1], for their model (a).

An important remark is that (1) has a constant of the motion with respect to the $x$-dependence of solutions. One verifies by integrations by parts

$$
\begin{aligned}
& \int_{-h / 2}^{h / 2} \mathrm{~d} y\left(w_{x}[\varphi, w]+\frac{1}{2} \varphi_{x}[w, w]\right)= \\
& =\frac{\mathrm{d}}{\mathrm{d} x}\left[\int_{-h / 2}^{h / 2} \mathrm{~d} y w_{x}\left(\varphi_{x} w_{y y}-\varphi_{y} w_{x y}\right)\right]
\end{aligned}
$$

whenever $w=\Delta w=0$ at $y= \pm h / 2$.

Multiplying (1a) by $w_{x}$ and (1b) by $\varphi_{x}$, integrating the result over $y$, and combining the result to make the cubic term disappear via (3), one gets after a few elementary manipulations

$$
\frac{\mathrm{d} K}{\mathrm{~d} x}=0
$$

where $K \equiv K^{\prime}+K^{\prime \prime}$ is the sought-after invariant. Here :

$$
\begin{aligned}
& K^{\prime}=\int_{-h / 2}^{h / 2} \mathrm{~d} y\left(M[w]+\frac{\lambda}{2} w_{x}^{2}-M[\varphi]\right) \\
& K^{\prime \prime}=\int_{-h / 2}^{h / 2} \mathrm{~d} y w_{x}\left(\varphi_{y} w_{x y}-\varphi_{x} w_{y y}\right)
\end{aligned}
$$

the quadratic functional $M$ being defined by

$$
M[\psi] \equiv \psi_{x} \psi_{x x x}-\frac{1}{2} \psi_{x x}^{2}-\psi_{x y}^{2}+\frac{1}{2} \psi_{y y}^{2}
$$

The computation of $K$ for periodic solutions as given by (2) is straightforward and yields, for the lowest relevant order :

$$
\begin{aligned}
K^{\prime} & =\frac{w_{0}^{2} b^{2}}{8} h(\varepsilon-16 b \delta)+\frac{w_{0}^{4} b^{4} h}{128} \\
K^{\prime \prime} & =-\frac{w_{0}^{4} b^{4}}{64} h
\end{aligned}
$$

where, again, $\delta \equiv a-b$ and $\varepsilon \equiv \lambda-\lambda_{\mathrm{c}}$.

Collecting together ( $4 a$ ) and (4b), and accounting for $w_{0}^{2} \simeq 8 \varepsilon / b^{2}$ (we assume that the selected wavenumber $a$ is such as $\delta \sim \varepsilon$, as in reference [1], and as verified a posteriori), one gets :

$$
K \simeq \frac{w_{0}^{2} b^{2} h}{8}\left(\frac{\varepsilon}{2}-16 b \delta\right)
$$

This allows one to fix one of the limits to the range of possible wavenumbers for half infinite solutions 
limited by a clamped edge. Near this clamped edge, the invariant $K$ reduces to

$\left.K\right|_{\partial}=\int_{-h / 2}^{h / 2} \mathrm{~d} y\left[-\left.\frac{1}{2}\left(w_{x x}^{2}-\varphi_{x x}^{2}+\varphi_{y y}^{2}\right)\right|_{\partial}\right]$.

From (6) and because $\varphi$ is vanishingly small near the clamped edge [7], $K$ must be negative in the bulk solution, and this implies, from $(5), \varepsilon / 32 b \leqslant \delta$.

The other limit to the wavenumbers for half infinite solutions is obtained as explained in reference [1] : one considers first the boundary layer joining the periodic solution far from the clamped edge to this edge. The corresponding form for the plate deviation is $w \simeq X(x) \sin a x \cos b y$, where the envelope function $X$ varies slowly on the spatial scale $h$ and where higher order terms are neglected. This envelope $X$ is the solution of [8]

$$
\varepsilon X-\frac{X^{3} b^{2}}{8}+4 X_{x x}=0 .
$$

For this particular problem the solution that satisfies the b.c. at the clamped edge $\left(w=w_{x}=0\right.$ at $\left.x=0\right)$ is

$$
X= \pm\left(\frac{8 \varepsilon}{b^{2}}\right)^{1 / 2} \tanh \left[x\left(\frac{\varepsilon}{8}\right)^{1 / 2}\right]
$$

This gives for $\left.w_{x x}\right|_{\partial}$ and $\left.\varphi_{x x}\right|_{\partial}$

$$
\begin{gathered}
{\left[\varphi \simeq \frac{X^{2}}{32}(\cos 2 a x-\cos 2 b y)\right]} \\
\left.w_{x x}\right|_{\partial}=\left.2 a X_{x}\right|_{\partial} \cos b y=\frac{2 a \varepsilon}{b} \cos b y
\end{gathered}
$$

and

$$
\left.\varphi_{x x}\right|_{\partial}=(1-\cos 2 b y) \frac{\left.X_{x}^{2}\right\rceil_{\partial}}{16}
$$

Once inserted into the expression of the invariant $K$ at the edge, one gets

$$
\left.K\right|_{\partial} \simeq-\varepsilon^{2} h \text {. }
$$

As in reference [1], this yields the other limit for the band of selected wavenumber. Once made equal to the expression for $K$, given in (5), this gives

$$
\varepsilon>\frac{32}{3} b \delta \text {. }
$$

All these considerations show that, near $\varepsilon=0_{+}$, the wavenumber of the buckling pattern is such that $a=b+\delta$, with

$$
\frac{\varepsilon}{32 b} \leqslant \delta \leqslant \frac{3 \varepsilon}{32 b}
$$

This is in agreement with the observation [4] that this wavelength tend to decrease as the load increases. Moreover, it may be readily shown by using results and methods of reference [1] that the various bifurcations producing this decrease of the wavelength follow the schema of reference [4]. As $\varepsilon$ increases at constant $L$, the buckling occurs first with a wavenumber (along $x$ ) close to the lower limit [in the $(\delta, \varepsilon)$ Cartesian plane $]$; that is, of order $b+\frac{3 \varepsilon}{32 b}$. Then (as $\lambda$ increases) it remains constant up to the crossing of the other limit; that is, at the line $\varepsilon=32 b \delta$. On this line, the buckling pattern changes by subcritical bifurcations, and this is accompanied by changes of symmetry for the overall solution, with respect to the middle of the rectangular plate. Then, as one smoothly increases the load, the $x$-wavenumber should remain close to the line $\varepsilon=32 b \delta$. If, on the contrary, the load decreases smoothly, this wavenumber should remain close to the lower limit

$$
\varepsilon=\frac{32 b \delta}{3} \text {. }
$$

The above analysis can equally be done when the long edges are clamped $(y= \pm h / 2)$, as are the short ones. The solution must be numerical however, as it implies transcendental equations.

Moreover, the present analysis shows how to get the adiabatic invariant (and thus the range of accessible wavenumbers) in cases more complicated than the ones considered in reference [1]. In a forthcoming publication we consider the case of the Taylor roll instability between rotating concentric cylinders (this is not spoiled by cross roll instabilities at the boundaries). In this last case the invariant can be computed almost as in the present one, except that the non linear advection term of the Navier-Stokes equation must be treated as the non linear term of model (b) of reference [1], since the invariant in this sort of problem is not exact, but only an adiabatic invariant.

\section{References}

[1] Pomeau, Y., Zaleski, S., « Wavelength selection in one dimensional cellular structures ", preprint DPh. T 81/80, June 1980, and submitted to J. Physique.

[2] In the elasticity literature, the non linear equations of this problem are often called the von Kármán equations, although Landau and Lifshitz [p. 79 in Theorie de l'élasticité, (ed. Mir, Moscou) 1967] attribute them to A. Foppl Vorlesungen über technishe Mechanik, Bd. 5, Leipzig (1907).

[3] It could be of experimental interest to consider long, but not necessarily, rectangular plates. They could be limited along the long side by wavy boundaries either with a regular or a random shape. In this last case, the first (linear) buckling mode should be localized, owing to the well known property of Anderson-Stieltjes localization in one dimensional random systems. The extension to two dimensional plates offers a possibility of analogic simulations of the $2 d$ localization problem. Furthermore, the non linear aspects of these buckling problems in complicated geometries certainly deserve some attention. 
[4] Schaeffer, D. and Golubitsky, M., Comm. on Math. Phys. 69 (1979) 209. In this paper references can be found to various aspects of this buckling problem. The authors thoroughly discuss the bifurcation of the buckling pattern as a function of plate length.

[5] The terms linear with respect to $w$ account for the elastic energy involved in the shear of the plate, although the Airy stress function accounts for the bulk compression (or expansion) of the plate parallel to its surface. The linear approximation (with respect to $w$ ) is valid when $w$ is smaller than the thickness of the plate. The form of the non linear terms in (1) implies that $w$ remains much smaller than the typical size of the plate, a realistic approximation in most cases. Moreover (1) is written in a dimensionless form, although the original equations are :

$$
\Delta^{2} \varphi+\frac{E}{2}[w, w]=0
$$

and

$$
\frac{l^{2} E}{12\left(1-\sigma^{2}\right)} \Delta^{2} w-[\varphi, w]=0
$$

where $l$ is the thickness of the plate, $E$ is the Young modulus, $\sigma$ the Poisson coefficient, and the stress tensor $\sigma$ is connected to the Airy function $\varphi$ by the formulae

$$
\sigma_{x x}=\varphi_{y y}, \sigma_{x y}=-\varphi_{x y} \text { and } \sigma_{y y}=\varphi_{x x} .
$$

[6] Sorokin, V. S., Priklad. Mat. i Mek. 28 (1954) 197.

[7] From (6), the contribution to $\left.K\right|_{\partial}$ quadratic in $\varphi$ is

$$
\int_{-h / 2}^{h / 2} \mathrm{~d} y\left(\varphi_{x x}^{2}-\varphi_{y y}^{2}\right) / 2,
$$

and this is negligible near $\varepsilon=0_{+}$compared to the other contribution, namely $-\frac{1}{2} \int_{-h / 2}^{h / 2} \mathrm{~d} y w_{x x}^{2}$. This last (and dominant) contribution is of order $\varepsilon^{2}$. To estimate the order of magnitude of $\varphi$ near the clamped edge, one notices first that $\varphi$ as given by the solution of $(1 b)$ is a quadratic functional of $w$, so that $\varphi$ is of order of the square of the amplitude of $w$. Actually $\varphi$ depends in a non local manner on $w$. One checks at once that when this amplitude of $w$ varies slowly, $\varphi$ depends on its value in a space domain of extent of order unity [this extent is, roughly speaking, the range of the operator $\Delta^{-2}$ needed to get $\varphi$ from (1b)]. Thus near the clamped edge $\varphi$ is order of the square amplitude of $w$ (i.e. $\left.X^{2}(x)\right)$ taken at some distance of order unity from the edge. Since $X$ vanishes at the edge as $\varepsilon x, \varphi$ is of order $\varepsilon^{2}\left[\sim(\varepsilon x)^{2}\right.$ with $\left.x \sim 1\right]$ near this edge. Accordingly, the contribution of $\varphi$ to $\left.K\right|_{\partial}$ is of order $\varepsilon^{4}$. Actually the derivatives with respect to $x$ and $y$ in $\varphi_{x x}$ and $\varphi_{y y}$ act on functions of $x$ and $y$ as $\cos 2 a x$ or cos $2 b y, \ldots$ so that $\varphi_{x x}^{2}$ and $\varphi_{y y}^{2}$ are of the same order of magnitude as $\varphi^{2}$. Thus near $\varepsilon=\left.0_{+} K\right|_{\partial} \simeq-\int_{-h / 2}^{h / 2} \mathrm{~d} y w_{x x}^{2} / 2$

[8] Segel, L. A., in Non equilibrium thermodynamics : variational techniques and stability, R. J. Donnelly, I. Prigogine and R. Hermann eds., (Univ. of Chicago Press) 1966 and Wesfreid, J., Pomeau, Y., Dubois, M., Normand, C. and Bergé, P., J. Physique 39 (1978) 725. In the present paper, we do not derive the envelope equations explicity, but the general method is well known and does not present any difficulty in the problem under consideration. 